\title{
Using Big Data to Discover Chaos in China's Futures Market During COVID-19
}

\author{
Lin Tie ${ }^{1}$, Bin Huang ${ }^{1}$, Bin $\operatorname{Pan}^{1}$ and Guang Sun ${ }^{1,2, *}$ \\ ${ }^{1}$ Hunan University of Finance and Economics, Changsha, 410205, China \\ ${ }^{2}$ University of Alabama, Tuscaloosa, AL 35487, USA \\ *Corresponding Author: Guang Sun. Email: simon5115@163.com \\ Received: 11 April 2021; Accepted: 27 May 2021
}

\begin{abstract}
COVID-19 was first reported in China and quickly spread throughout the world. Weak investor confidence in government efforts to control the pandemic seriously affected global financial markets. This study investigated chaos in China's futures market during COVID-19, focusing on the degree of chaos at different periods during the pandemic. We constructed a phase diagram to observe the attractor trajectory of index futures (IFs). During the COVID-19 outbreak, overall chaos in China's futures market was increasing, and there was a clear correlation between market volatility and the macroenvironment (mainly government regulation). The Hurst index, calculated by rescaled range $(\mathrm{R} / \mathrm{S})$ analysis, was 0.46 . The price and return of IFs showed long-term correlation and fractal characteristics; the relevant dimensions of the futures market were 2.17. Overall, under the influence of an emergency (COVID-19), chaos in China's financial market intensified, creating a need for timely government intervention and macrocontrol of the market. This study's findings can help improve the government's understanding of the phenomenon of financial chaos caused by emergencies. This study also provides theoretical guidance for controlling financial chaos and maintaining healthy economic development when faced with similar events in the future.
\end{abstract}

Keywords: Chinese futures market; COVID-19; chaos; Lyapunov index; Hurst index

\section{Introduction}

Chaos in financial systems arises from instability in their internal operations. External manifestations of such chaos include sharp, abnormal fluctuations and financial crises. Moreover, inefficient market-resource allocation can greatly affect the economy and society. Therefore, evidence of chaos can have significant effects on regulators and on short-term trading strategies.

Although China's financial market has developed rapidly, it has an incomplete and unbalanced structure, and it has gradually shown the characteristics of chaos. Studies of the Shanghai Stock Exchange have found that China's stock market has significant chaotic features, with a chaotic attractor dimension of 2-3 [1,2]. The futures market is a central part of China's financial market. Some studies have taken commodity futures as the research object to study China's futures market.

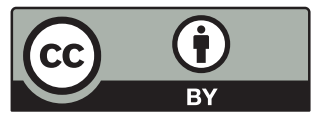

This work is licensed under a Creative Commons Attribution 4.0 International License, which permits unrestricted use, distribution, and reproduction in any medium, provided the original work is properly cited. 
These include agricultural product futures, such as soybeans, wheat, and mung beans [3-6], and bulk metal commodity futures, such as gold, silver, copper, and aluminum [5-7]. Fewer studies, however, have focused on financial futures in China, and even fewer have specifically investigated chaos in the futures market in the context of emergencies (e.g., epidemics).

COVID-19 quickly spread throughout China in late 2019 and propagated globally. Since then, numerous studies have investigated the various effects of COVID-19 [8-15]. The pandemic has had far-reaching effects on the global economy. In March 2020, for example, the Dow Jones Industrial Average fell by nearly $40 \%$ [16]. International oil prices fell sharply, and markets such as gold and bonds were affected as well [17]. Panic in financial markets gave rise to irrational decision-making [17]. Early in the pandemic, McKibbin et al. [18] used a DSGE/CGE model to examine the effects of seven possible scenarios and determined that a global pandemic would have enormous negative effects on the world economy; impoverished countries in particular could face major devastation. Khan et al. [19], meanwhile, studied COVID-19's effects on stock markets and found that, in the short-window period, global stock markets suffered enormous negative effects while in the long-window period, the Shanghai Composite Index saw a recovery. Almehmadi [20] developed an AI system to study the effect of public opinion on the stock market and used the Dow Jones Industrial Average and Saudi Stock Exchange to test it, achieving an accuracy rate of $99.71 \%$. With regard to futures, various studies have investigated the pandemic's effects on gold [21], energy [22], agricultural products, crude oil [23,24], and other futures.

At the beginning of the COVID-19 outbreak, investors in China were initially optimistic, but after Wuhan was locked down on January 23, 2020, their confidence was shaken. In early February, China's financial market saw an unprecedented plunge. However, from February 4 to February 21, 2020, as the government took effective COVID-19 control measures, investor confidence rebounded, and the market improved somewhat. Yet, in March 2020, COVID-19 began to spread out of control overseas, the WHO declared it a pandemic [25], and investor confidence plummeted. The US stock market fell sharply, and the global linkage effect led to a sharp decline in China's stock market as well. The Chinese futures market was also affected, causing violent fluctuations. During the pandemic's development, the oscillations in China's futures market contained various high-dimensional information, which underwent a series of changes and was ultimately reflected in futures prices.

This study focused on chaos in China's futures market after the COVID-19 outbreak. It mainly contributes to the literature on the effect of emergencies (pandemics) on economic chaos in China. Taking China's futures market as the research object, we selected price series of index futures (IFs) before and after the main development period of the pandemic in China (November 1, 2019, to May 31, 2020) to establish a time series. The next section introduces the methods. Then, Section 3 describes the data set and the empirical results; we also use the concept of chaos to examine the effect of "big events" during COVID-19 on futures market chaos in China. Section 4 summarizes the paper and discusses the limitations.

\section{Method}

This study used big data to simulate chaos in China's futures market during COVID-19. The methods included a phase diagram, maximum Lyapunov exponent, correlation dimension, and Hurst exponent. Fig. 1 shows the research route of the study.

We reconstructed the phase space of IF exponential time series, calculated the most prominent Lyapunov exponent of the series, and used visualization techniques to visualize chaotic attractors. 
Meanwhile, the fractal dimension and Hurst index were used to judge the chaotic characteristics of the futures market.

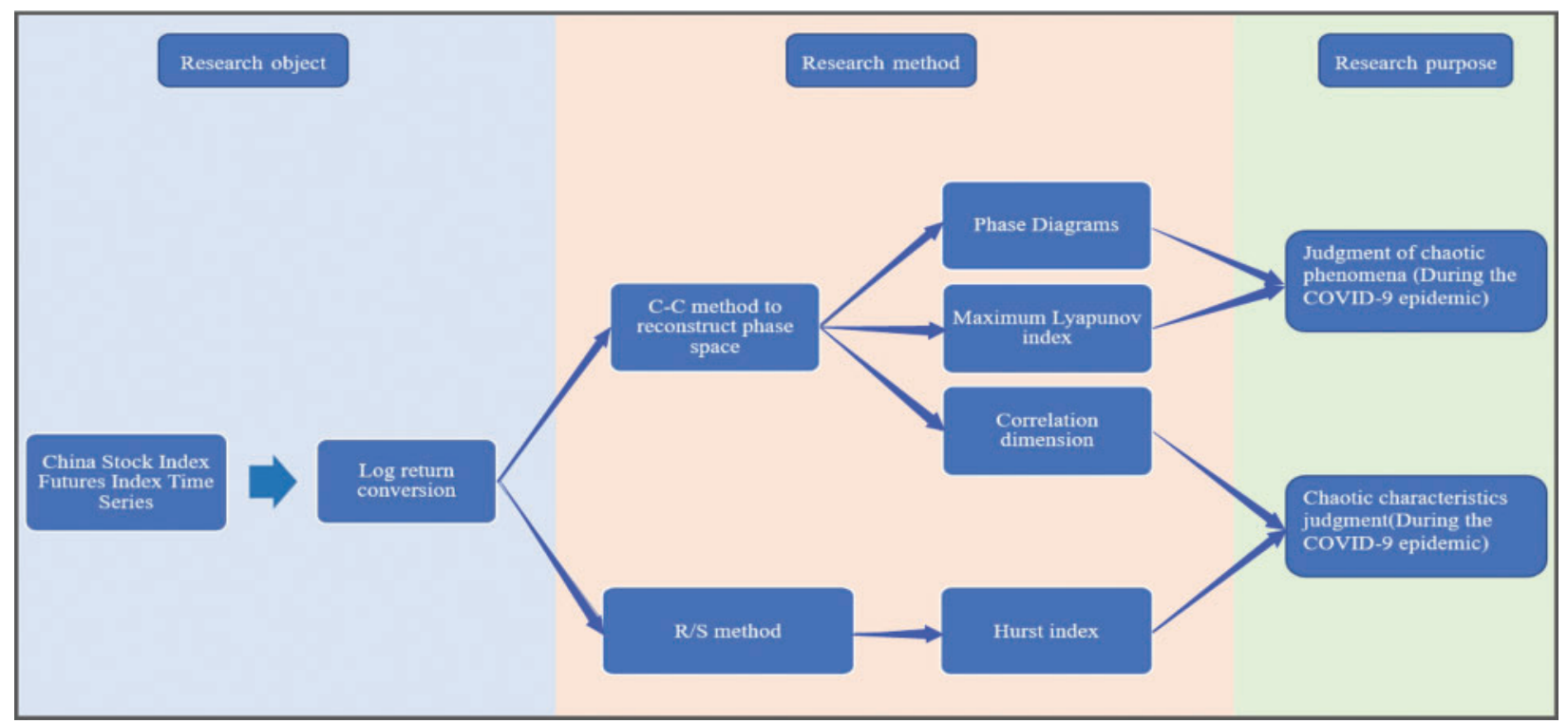

Figure 1: Research route

\subsection{Phase-Space Reconstruction and C-C Method}

Time-series phase diagrams can be used to analyze changes in a nonlinear system with a time state; this can reflect the system attractor's spatial structure. A chaotic attractor of the highdimensional region is first proposed in the phase space to restore the chaotic system. The principle here is that the evolution of any component in a system depends on the interaction between other members. Information about these related components will be retained during the development of any element. We can extract and restore the original law of the system from the time-series data of any component, which is a trajectory in high-dimensional space. The revolution reflects the regularity of the chaotic system. The evolution of a chaotic system will eventually fall into a specific course, which is the chaotic attractor.

We used the $\mathrm{C}-\mathrm{C}$ algorithm to calculate time-lag parameter $\mathrm{t}$ and embed dimension $\mathrm{m}$ of the IF time series [26]. The $\mathrm{C}-\mathrm{C}$ method is based on statistical results, and it obtains time-delay parameter $\mathrm{t}$ and time-window $\tau \mathrm{m}$ through correlation integration. Then, the embedding dimension is received through the relationship between the time window and the embedding dimension as shown in Fig. 2.

Suppose there is a chaotic time series, $X=\left\{X_{i} \mid i=1,2,3,4, \ldots, N\right\}$; the embedding dimension is $\mathrm{m}$, the time-delay parameter is $\mathrm{t}$, and the reconstructed phase space $X=\left\{X_{i}\right\}$ is the point of the reconstructed phase space. Decompose the time series into t nonoverlapping subsequences as follows:

$X_{1}=\left\{X_{i} \mid i=1, t+1, \ldots, N-t+1\right\}$,

$X_{2}=\left\{X_{i} \mid i=2, t+2, \ldots, N-t+2\right\}$, 
$X_{t}=\left\{X_{i} \mid i=1, t+1, \ldots, N-t+1\right\}$,

where $\mathrm{N}$ is an integer multiple of $\mathrm{t}$. The statistics are defined, and the block average strategy is adopted:

$S_{1}(m, N, r, t)=C(m, N, r, t)-C^{m}(1, N, r, t)$,

$S_{2}(m, N, r, t)=\frac{1}{t} \sum_{s=1}^{t}\left[C_{s}\left(m, \frac{N}{t}, r, t\right)-C_{s}^{m}\left(1, \frac{N}{t}, r, t\right)\right]$,

where $C$ is the correlation integral, $m$ is the number of phase points, $r$ is the radius of the field, and $t$ is the Euclidean distance from coordinate $\mathrm{I}$ to coordinate $\mathrm{j}$ in the phase space. Let $\mathrm{N} \rightarrow \infty$ have

$S_{2}(m, r, t)=\frac{1}{t} \sum_{s=1}^{t}\left[C_{s}(m, r, t)-C_{s}^{m}(1, r, t)\right]$.

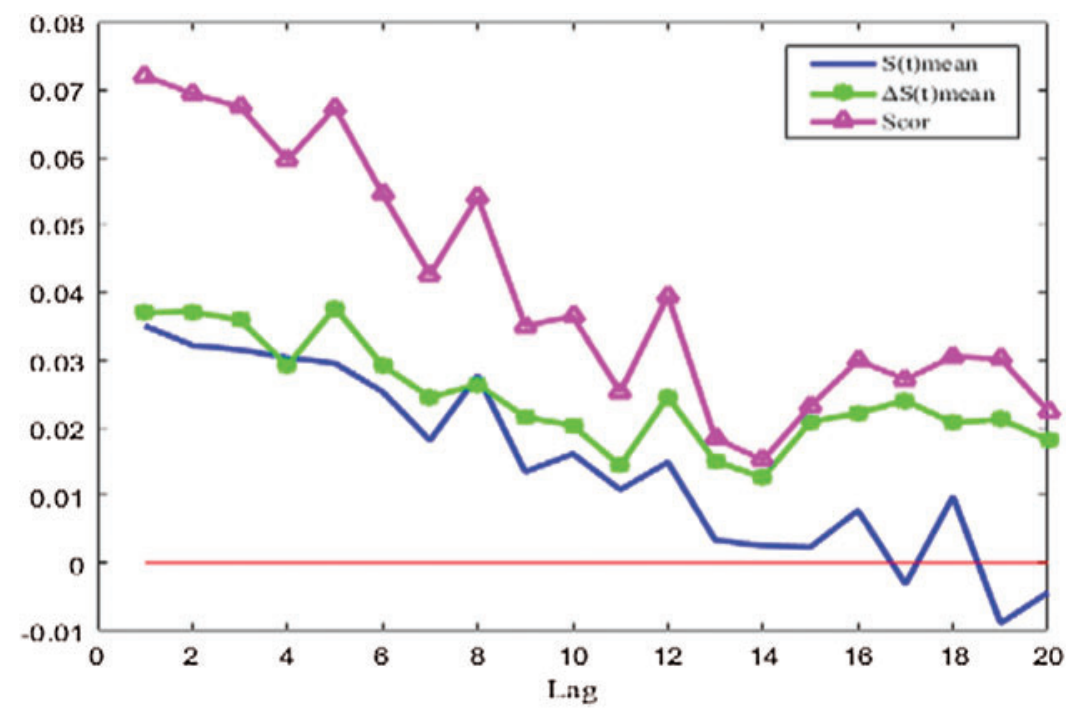

Figure 2: IF index time-series $\mathrm{C}-\mathrm{C}$ method

If the time series is independent and identically distributed, then for a fixed-delay parameter $\mathrm{t}$ and embedding dimension $\mathrm{m}, \mathrm{N} \rightarrow \infty$. For any $\mathrm{r}, S_{2}(m, r, t)$ is always equal to zero. However, the actual time series is not infinitely long. There are correlations between elements, which leads to $S_{2}(m, r, t) . S_{2}(m, r, t) \sim \mathrm{t}$ reflects the lag correlation of the time series, imitating the principle of the lag correlation method for finding the time-delay parameters. The optimal time-delay $\mathrm{d} \tau$ can be the first of $S_{2}(m, r, t) \sim \mathrm{t}$ zero points; or, take $S_{2}(m, r, t) \sim \mathrm{t}$ for all time points with the smallest difference between radius $r$, which means the issues in the reconstructed phase space are closest to uniform distribution. Rebuild the attractor orbit to make it fully expand in the phase space. Then, choose the two largest and smallest radii and define the difference:

$\Delta S_{2}(m, t)=\max \left\{S_{2}\left(m, r_{j}, t\right)\right\}-\min \left\{S_{2}\left(m, r_{j}, t\right)\right\}$. 
This measures the maximum deviation of $S_{2}(m, r, t) \sim \mathrm{t}$ to the radius $\mathrm{r}$ of all fields, reflecting the time-series lag correlation. In summary, the optimal time-delay parameter can be the first zero point of $S_{2}(m, r, t) \sim \mathrm{t}$ or the first local minimum point of $\Delta S_{2}(m, t) \sim \mathrm{t}$. When $\Delta S_{2}(m, t)$ takes the minimum value, we can calculate the phase space-time window; then, we can obtain the embedding dimension $m=1+\frac{\tau m}{t}$.

Based on the above principles, we find that the first local minimum occurs when the delay parameter is 4 . Accordingly, we selected 4 as the best delay parameter.

\subsection{Most Prominent Lyapunov Exponent}

In practical application, the Lyapunov exponent is an important index for quantitatively measuring a system's dynamics. Its geometric meaning is to quantify the exponential divergence of the initial closed-loop orbit and the amount of chaos in the nonlinear system. It represents the adjacent orbits of the system in the phase space - that is, the average exponential rate of convergence or divergence. (The Lyapunov exponent is greater than 0 . This means that no matter how small the initial trajectory distance in the system's phase space is, it will increase exponentially with the evolution of time and is ultimately unpredictable. It is a chaotic phenomenon.)

Assuming there is a time series, the time-delay parameter is $\mathrm{t}$, the embedding dimension is $\mathrm{m}$, and the phase space of the reconstructed time series is $Y=\left\{Y_{j}, j=1,2,3, \ldots, M\right\}$. Then, find the nearest neighbor of each point in the phase space and limit the short separation:

$d_{j}(0)=\min _{\wedge j}|| Y_{j}-X_{\wedge j+1} \|, \quad|j-\wedge j|>p$.

For each $Y_{j}$ in the phase space, calculate the distance of discrete time steps of the adjacent point pair:

$d_{j}(i)=\left|Y_{j+1}+Y_{\wedge j+1}\right|$,

$i=1,2, \ldots, \min (M-j, M-\wedge j)$.

For each $i$, find the average of all $\mathrm{j}$; namely,

$y(i)=\frac{1}{q \Delta t} \sum_{i=1}^{q} \ln d_{j}(i)$,

where $\mathrm{q}$ is the number of nonzeros, and the regression line is made using the least-squares method. The slope of the line is the largest Lyapunov exponent. In 1983, Gribo proved that the time series with the largest Lyapunov exponent greater than 0 is chaotic. Therefore, we do not need to calculate all of the Lyapunov exponents; we only need to figure out whether the most prominent Lyapunov exponent is greater than 0 .

The small data volume method is relatively reliable for calculating the most prominent Lyapunov exponent of a time series [27]. The operation is simple, and the calculation volume is small. These characteristics have excellent robustness to the actual financial time series (i.e., the total data volume is small, and the time-series noise is considerable).

\subsection{Correlation Dimension}

The existence of strange attractors is an essential feature of chaotic dynamic systems. The divergence of the attractor in space is an irregular movement in a limited area, and we used the 
fractal dimension to describe the singularity of its activity. For the phase space, we define the distance between these points as the maximum component difference between the two points:

$\left|y_{i}-y_{j}\right|=\max _{1 \leq k \leq m}\left|y_{i k}-y_{j k}\right|$.

If the distance is less than the positive number of the field's radius, it is called the associated point. Assuming there is such a point in the phase space, calculate the correlated topics' logarithm. The proportion of all possible species pairings becomes the correlation integral; namely,

$C(r)=\frac{1}{N^{2}} \sum_{i, j=1}^{N} \theta\left(r-\left|y_{i}-y_{j}\right|\right)$.

Select an appropriate choice so that there is a specific interval, where D is the correlation dimension. If the correlation dimension calculated by the system's attractor is a fractal dimension, the system attractor appears in a chaotic state when it moves. The fractal dimension of the phase space depends on the complexity of the system. All variables in the phase space that are higher than the next integer of the fractal size are the dynamic system model's minimum dynamic variables.

\subsection{Hurst Index}

The Hurst index is a parameter for judging whether a time series is dependent on time. In this study, we used rescaled range $(\mathrm{R} / \mathrm{S})$ to calculate the Hurst index of the time series and calculate the fractal dimension of the string through the Hurst index.

The main steps of the R/S method are as follows: Return the logarithm of the sequence length divided into subsequences, and the interval length of each subset is xai. Furthermore, calculate the mean value of each subset a, denoted as:

$\bar{X}_{a}=\frac{1}{n} \sum_{j=1}^{n} X_{a j}, \quad \mathrm{a}=1,2, \ldots, \mathrm{A}$.

Choose a subset arbitrarily and calculate the cumulative and standard deviations of the firsts points one by one:

$y_{a j}=X_{a j}-\bar{X}_{a}$,

$Z_{a j}=\sum_{k=1}^{j} y_{a k}$

Calculate the fluctuation range $\mathrm{R}$ of subset a and the standard deviation $\mathrm{S}$ of each subset a pair sequence:

$R_{a}=\max \left(Z_{a j}\right)-\min \left(Z_{a j}\right), \quad \mathrm{a}=1,2,3, \ldots, \mathrm{A} ; \mathrm{j}=1,2, \ldots, \mathrm{n}$,

$S_{a}=\sqrt{\frac{1}{n-1}} \sum_{j=1}^{n}\left(X_{a j}-\bar{X}_{a}\right)^{2}, \quad \mathrm{a}=1,2,3, \ldots, \mathrm{A} ; \quad \mathrm{j}=1,2, \ldots, \mathrm{n}$. 
The standard deviation $\mathrm{S}$ is used for each subset to standardize the fluctuation range $\mathrm{R}$ and obtain the R/S rescaling range. Multiple experiments can be performed by selecting different $n \mathrm{~s}$ :

$R S_{a}=\frac{R_{a}}{S_{a}}$

$\left(\frac{R}{S}\right)_{n}=\frac{1}{A} \sum_{i=1}^{A} R S_{a}$.

Calculate the Hurst index as follows:

$\log \left(\frac{R}{S}\right)=H \log (n)+\log (K)$,

where the $\mathrm{K}$ is a constant.

\section{Results Analysis}

The data for this study came from the China Financial Futures Exchange (http://www.cffex. com.cn/). The study period was November 1, 2019, to May 31, 2020 (the main period of the COVID-19 outbreak in China). We performed log-trend elimination on the collected futures timeseries data and calculated the logarithmic return rate after eliminating the trend. Figs. 3 and 4 shows the results.

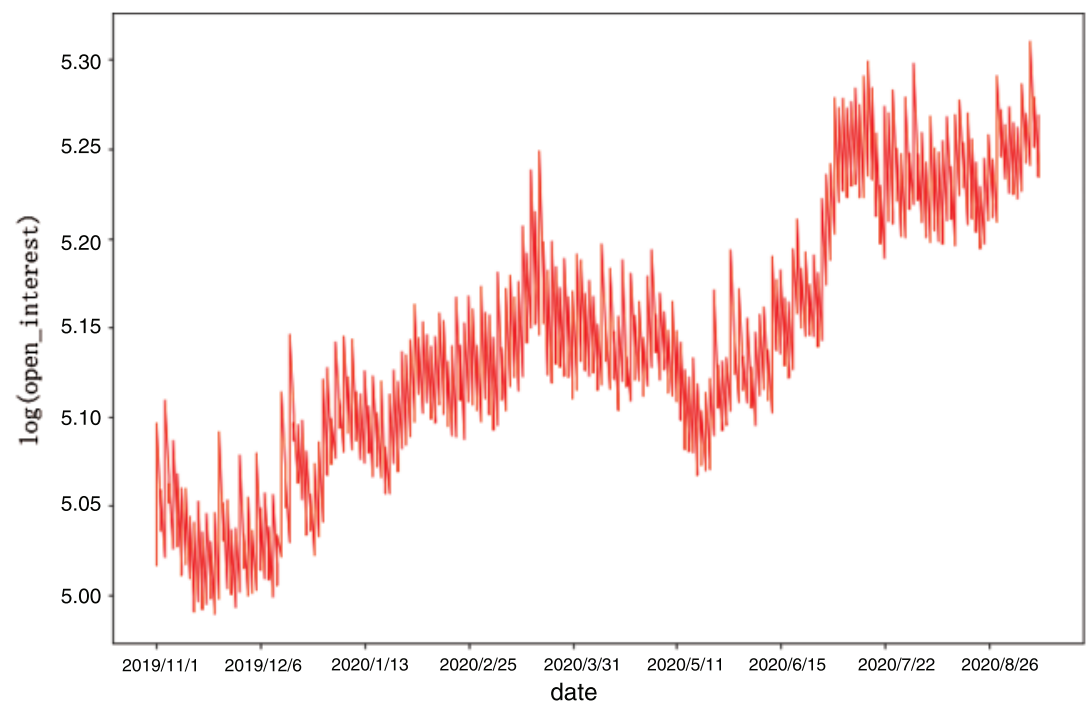

Figure 3: Logarithm of daily settlement IF prices

Fig. 3 shows the daily closing prices of IFs. We obtained the logarithmic time series through logarithmic processing.

After the trend-elimination processing of the sequence in Fig. 3, we find that under the influence of COVID-19, the futures market still showed an upward trend, but the logarithmic yield showed random characteristics. 


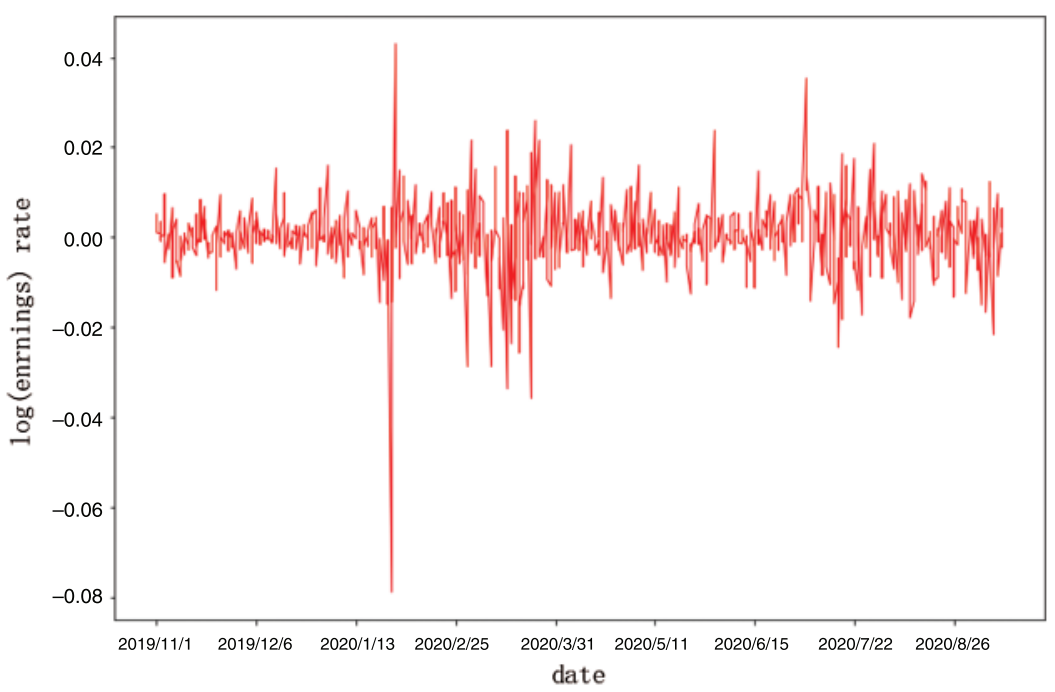

Figure 4: Log returns

\subsection{Phase Diagram of IFs}

We reconstructed the phase space of the financial time series and constructed the phase diagram using the visualization method. The phase diagram was used to determine the chaotic characteristics of IF time series. First, it was necessary to confirm the phase-space reconstruction to determine the time-delay parameter $\mathrm{t}$ and the embedding dimension $\mathrm{m}$. The best embedding time $\mathrm{t}$ we chose in Section 2.1 was 4 , and the best embedding dimension $\mathrm{m}$ was 4.

As shown in Fig. 5, in the reconstructed high-dimensional phase space, the IF time-series data were not randomly distributed like random white noise but repeatedly entangled in local areas and wrinkles. In the end, it formed a strange attractor. Its trajectory was neither a reciprocating periodic function nor a random movement without regularity, showing a more significant chaotic phenomenon.

\subsection{Chaos in the Futures Market in Different Periods of the Pandemic}

For a chaotic economic system, its Lyapunov exponent is the system's fractal dimension, and $\mathrm{g}$ is the range:

$g=\max (F(I))-\min (F(I))$.

Then, the vector is

$a=(\lambda, d, g)$.

This is the system's chaotic degree vector, where $\lambda$ is the largest Lyapunov exponent of the system.

We split the data to examine chaos in the futures market during different periods. Experiments showed that the overall maximum Lyapunov exponent $\lambda$ from November 2019 to May 2020 finally converged to 0.775 . The system's fractal dimension d was 2.173 , and the logarithmic return range g was 0.1217 . Tab. 1 shows the period divisions.

From Tab. 1, we can derive the following conclusions:

1. Chaos existed in China's futures market. 

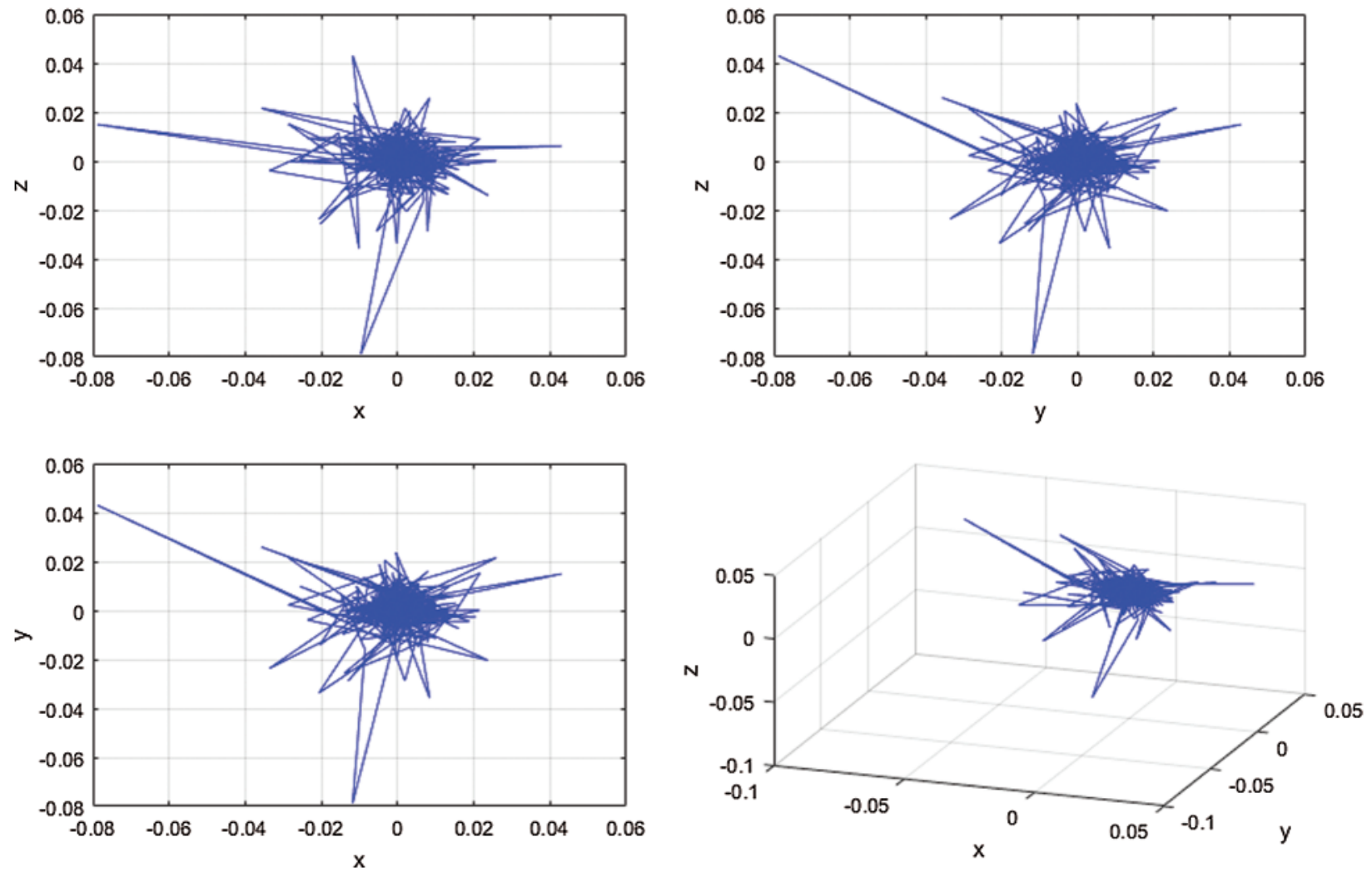

Figure 5: Sixty-minute time-series phase space of IFs

Table 1: Chaos degree of IFs in different periods

\begin{tabular}{llll}
\hline Sample interval & $\lambda$ & $\mathrm{d}$ & $\mathrm{g}$ \\
\hline $2019.11-2020.02$ & 0.7517 & 1.955 & 0.121764 \\
$2020.01-2020.03$ & 0.2333 & 2.092 & 0.121764 \\
$2020.02-2020.04$ & 0.4133 & 2.102 & 0.121764 \\
$2020.03-2020.05$ & 0.411 & 1.731 & 0.061671 \\
$2020.04-2020.06$ & 0.6894 & 1.736 & 0.037273 \\
$2020.05-2020.07$ & 0.633 & 1.408 & 0.059755 \\
$2019.05-2020.05$ & 0.775 & 2.173 & 0.121764 \\
\hline
\end{tabular}

2. After the outbreak, the degree of chaos in the futures market showed an upward trend over time, and the overall degree of chaos was higher than the local ones.

3. There were large fluctuations in China's futures market and the macroenvironment (mainly government intervention: when the government exhibited better control of the pandemic, investors had more confidence; otherwise, they were less willing to invest). Tab. 2 reveals more obvious correlations. 
Table 2: Relationship between IF fluctuations and changes in the macroenvironment

\begin{tabular}{llll}
\hline Time & Peak & Index & Event \\
\hline January 2020 & Low & 3961.8 & COVID-19 outbreak in China \\
February 2020 & Peak & 4192.6 & Government responded quickly to control the epidemic \\
March 2020 & Low & 3477.0 & Linkage effect caused by the crash of US stocks \\
April 2020 & Peak & 3911.8 & Pandemic in China was largely contained \\
\hline
\end{tabular}

\subsection{Hurst Index}

As shown in Fig. 6, the Hurst index of the logarithmic time series of IFs calculated by R/S analysis was 0.46 , which is lower than 0.5 but close to 0.5 . This indicates that volatility in the futures market had a specific long-term correlation. In other words, Chinese IFs did not show random-walk characteristics, but the overall trend was still nonlinear. Reflecting this conclusion, the calculated correlation scale of IFs was not 0 . The reason for this could be that, affected by the pandemic, many kinds of nonlinear information originally existed in the market. People responded to this information in a correspondingly nonlinear way with regard to decision-making behavior and investment willingness. The result was that prices ultimately reflected this nonlinear information. In other words, the trading activities of the entire market were reflected in the IFs, making the time series of the IFs also show nonlinear characteristics.

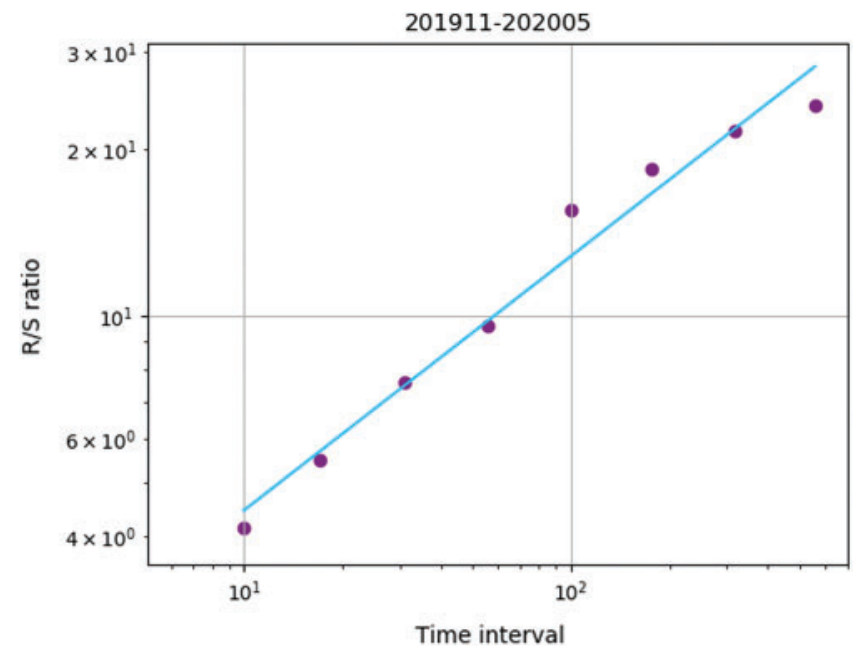

Figure 6: R/S method for calculating the Hurst exponent fitting curve

\section{Conclusion}

The sudden global public health event of COVID-19 at the end of 2019 increased nonlinearity in global economic systems and produced chaos. The differing strategies of various countries in response to the pandemic affected the global economy in a nonlinear manner. China's financial system became unstable, and in February and March 2020, its futures market experienced two sharp declines, which had negative effects on economic development and social stability. 
This study used the $\mathrm{C}-\mathrm{C}$ method to reconstruct the phase space of the time series. Moreover, we observed the chaotic attractor trajectory of China's futures market in the phase diagram. The maximum Lyapunov exponent of the system was greater than 0 and therefore had a chaos effect. The overall level of chaos in China's futures market increased during the pandemic; this phenomenon was related to intervention by the macroenvironment.

During the pandemic, the fractional dimension of the attractor of China's futures market was 2.173. The fractal dimension also revealed that, for financial markets, we need to consider many uncertain factors, such as human psychological factors, decision-making, and information/information asymmetry. The market and its movement are still dominated by factors that are relatively difficult to measure, and the macroenvironment (COVID-19 in this case) can magnify these uncertainties. The fractal dimension is the smallest variable of the system-measurement factor. This means that during a pandemic, the futures market needs at least three dynamic variables, which we believe should be investor expectations, price estimates, and market liquidity.

The Hurst index of China's futures market was 0.46 . This means that the market had fractal characteristics - namely, self-similarity and long-term correlation. Self-similarity refers to recursion and pattern similarity while long-term correlation means there is no significant correlation between past and future. This feature causes the market to have certain risks.

External performance refers to the continuous rise and fall of futures prices. Volatility clustering makes the large-scale fluctuation of endogenous variables concentrate on the period while small-scale undulation occurs during a short period. Although chaos and randomness increased in China's financial trading market after COVID-19, investors could still profit from it. Their ability to realize profits was dependent on investors' understanding of stock-market characteristics.

This study has some limitations. First, we did not consider how the linkage effect between global stock markets affected chaos in China's futures market during COVID-19. Second, because of the intensification of chaos in the futures market caused by an emergency, we did not conduct a deeper exploration of the subsequent development of the market. The next step will be to dig deeper into how financial market chaos affects subsequent market development and find ways to control such chaos.

Funding Statement: This research was funded by This work was supported by the National Natural Science Foundation of China (No. 72073041); Open Foundation for the University Innovation Platform in Hunan Province (No. 18K103); 2011 Collaborative Innovation Center for Development and Utilization of Finance and Economics Big Data Property, Universities of Hunan Province, Open Project (Nos. 20181901CRP03, 20181901CRP04, 20181901CRP05); 2020 Hunan Provincial Higher Education Teaching Reform Research Project (Nos. HNJG-2020-1130, HNJG-2020-1124); and 2020 General Project of Hunan Social Science Fund (No. 20B16).

Conflicts of Interest: The authors declare that they have no conflicts of interest to report regarding the present study.

\section{References}

[1] D. Luo, "Chaotic characteristics of the SSE composite index time series," in Proc. ICEEE, Zhengzhou, Henan, CHN, pp. 1-4, 2010.

[2] P. Zhen, Y. Zeng, J. Zhou and L. Lv, "Chaotic analysis and dimension reduction of Shanghai composite index time series," in Proc. ICM, Beijing, CHN, pp. 4-8, 2017. 
[3] L. Y. He and S. P. Chen, "Multifractal detrended cross-correlation analysis of agricultural futures markets," Chaos, Solitons \& Fractals, vol. 44, no. 6, pp. 355-361, 2011.

[4] L. Y. He, S. D. Zhou and C. H. Xu, "Fractal and multifractal features in China's agricultural commodity futures prices," Chinese Agricultural Science Bulletin, vol. 24, no. 3, pp. 481-485, 2008.

[5] K. C. Chan, H. G. Fung and W. K. Leung, "Daily volatility behavior in Chinese futures markets," Journal of International Financial Markets Institutions and Money, vol. 14, no. 5, pp. 491-505, 2004.

[6] C. Q. Ma, C. Liu and H. Q. Li, "Study on nonlinear fluctuation characteristics in Shanghai metal futures market," The Theory and Practice of Finance and Economics, vol. 30, no. 1, pp. 36-40, 2009.

[7] M. A. Khan, S. Abbas, K. M. Khan, A. M. and A. Rehman, "Intelligent forecasting model of covid-19 novel coronavirus outbreak empowered with deep extreme learning machine," Computers, Materials \& Continua, vol. 64, no. 3, pp. 1329-1342, 2020.

[8] R. Ünlü and E. Naml,, "Machine learning and classical forecasting methods based decision support systems for covid-19," Computers, Materials \& Continua, vol. 64, no. 3, pp. 1383-1399, 2020.

[9] Y. Zhang, J. Cheng, Y. Yang, H. Li, X. Zheng et al., "Covid-19 public opinion and emotion monitoring system based on time series thermal new word mining," Computers, Materials \& Continua, vol. 64, no. 3, pp. 1415-1434, 2020.

[10] M. Naveed, D. Baleanu, M. Rafiq, A. Raza, A. H. Soori et al., "Dynamical behavior and sensitivity analysis of a delayed coronavirus epidemic model," Computers, Materials \& Continua, vol. 65, no. 1, pp. 225-241, 2020.

[11] W. Alhakami, A. Binmahfoudh, A. Baz and M. Tarique, "Atrocious impinging of covid-19 pandemic on software development industries," Computer Systems Science and Engineering, vol. 36, no. 2, pp. 323-338, 2021.

[12] A. Baz and H. Alhakami, "Fuzzy based decision making approach for evaluating the severity of covid19 pandemic in cities of kingdom of saudi arabia," Computers, Materials \& Continua, vol. 66, no. 2, pp. 1155-1174, 2021.

[13] S. Ketu and P. K. Mishra, "A hybrid deep learning model for covid-19 prediction and current status of clinical trials worldwide," Computers, Materials \& Continua, vol. 66, no. 2, pp. 1896-1919, 2021.

[14] M. Cunill, M. A. Andreu, B. S. Ferrer and J. P. Masó, "The impact of COVID-19 on Spanish health professionals: A description of physical and psychological effects," International Journal of Mental Health Promotion, vol. 22, no. 3, pp. 185-198, 2020.

[15] S. Azam, N. Ahmed, A. Raza, M. S. Iqbal and M. Rafiq, "Numerical analysis of novel coronavirus (2019-ncov) pandemic model with advection," Computers, Materials \& Continua, vol. 67, no. 3, pp. 29332953, 2021.

[16] F. Imbert and Y. Li, "Dow falls more than 30 points, but Nasdaq ekes out fifth straight gain. USA: CNBC, 2020. [Online]. Available: https://www.cnbc.com/2020/06/17/stock-market-futures-open-to-closenews.html.

[17] J. Wiebe, T. Wilson and C. Cardie, "Annotating expressions of opinions and emotions in language," Language Resources and Evaluation, vol. 39, no. 2, pp. 165-210, 2005.

[18] W. Mckibbin and R. Fernando, "The global macroeconomic impacts of covid-19: Seven scenarios," Asian Economic Papers, vol. 20, no. 2, pp. 1-30, 2021.

[19] K. Khan, H. Zhao, H. Zhang, H. Yang and A. Jahanger, "The impact of COVID-19 pandemic on stock markets: An empirical analysis of world major stock indices," Journal of Asian Finance Economics and Business, vol. 7, no. 7, pp. 463-474, 2020.

[20] A. Almehmadi, "Covid-19 pandemic data predict the stock market," Computer Systems Science and Engineering, vol. 36, no. 3, pp. 451-460, 2021.

[21] L. Yarovaya, R. Matkovskyy and A. Jalan, "The effects of a 'black swan' event (COVID19) on herding behavior in cryptocurrency markets: Evidence from cryptocurrency USD, EUR, JPY and KRW markets," SSRN Electronic Journal, 2020, [Online]. Available: https://ssrn.com/abstract $=3586511$.

[22] D. Aloui, S. Goutte, K. Guesmi and R. Hchaichi, "COVID 19's impact on crude oil and natural gas S\&P GS Indexes," Working Paper, 2020. [Online]. Available: https://halshs.archives-ouvertes.fr/halshs02613280/document. 
[23] J. Wang, W. Shao and J. Kim, "Analysis of the impact of COVID-19 on the correlations between crude oil and agricultural futures," Chaos, Solitons \& Fractals, vol. 136, pp. 109896, 2020.

[24] A. Sharif, C. Aloui and L. Yarovaya, "COVID-19 pandemic, oil prices, stock market, geopolitical risk and policy uncertainty nexus in the US economy: Fresh evidence from the wavelet-based approach," International Review of Financial Analysis, vol. 70, pp. 101496, 2020.

[25] WHO Director-General's, "WHO Director-General's opening remarks at the media briefing on COVID-19-11 March 2020," The media briefing on COVID-19, 2020. [Online]. Available: https://www.who.int/director-general/speeches/detail/who-director-general-s-opening-remarks-at-the-mediabriefing-on-covid-19-20-march-2020.

[26] H. S. Kim, R. Eykholt and J. D. Salas, "Nonlinear dynamics, delay times and embedding windows," Physica D: Nonlinear Phenomena, vol. 127, no. 1, pp. 48-60, 1999.

[27] D. Kugiumtzis, "State space reconstruction parameters in the analysis of chaotic time series-The role of the time window length," Physica D: Nonlinear Phenomena, vol. 95, no. 1, pp. 13-28, 1996. 